\title{
Kinematics Smulation Of Six Degrees Freedom Robot Car Skylight Detection Based On ADAMS
}

\author{
Yamei Liu ${ }^{1, a}$, Jing Liu ${ }^{2, b}$,Yahui Bian ${ }^{3, c}$, Yongpan $\mathrm{Li}^{4, \mathrm{~d}}$ \\ ${ }^{1,2,3}$ Changchun University of Technology, China \\ ${ }^{4}$ Technical Center of Great Wall Motor Co., Ltd. Automotive Engineering Technology Research \\ Center, Baoding City Hebei Province China \\ aliuyamei2004@126.com, ${ }^{b} 932632468 @ q q . c o m,{ }^{c} 1506076566 @ q q . c o m,{ }^{d}$ liyongpan369@163.com
}

Keyword: ADAMS, Kinematics simulation, Sealing

\begin{abstract}
A function of automotive exteriors, automobile skylight from the early applied to high-end models, development up to now, have become more popular in the car at all levels. Enclosed is the priority study of automobile skylight, the sealing test is becoming more and more attention, in modern society, people's inspection work gradually replaced by robotic. For multi degree of freedom series robot, Kinematics positive solutions need a large number of complex matrix operations, big workload and error-prone. We can greatly simplify the calculation work by the dynamic simulation analysis software ADAMS of mechanical system, saves the cost of production, the kinematics and dynamics performance of the robot also can visually displayed by simulation and data chart .In the paper,we take Es165d type 6-dof industrial robot as the prototype, use the ADAMS simulation software to establish the virtual prototype of the robot to simulation kinematics study
\end{abstract}

\section{Introduction}

Introduction To Automobile Skylight. The skylight installed on the car roof can widen our vision, make the air circulation inside the car. The most important of automobile skylight is its sealing when need closing, it can't effectively block rain, snow, wind, driving resistance will also increase if sealing is not good. Therefore, it is necessary to detect seal of skylight [1]. We place car skylight to be detected in fixed detection platform in order to implement automobile skylight detection(skylight testing point distribution is shown in Figure 1), by controlling the movement of the robot, promoting the detection sensor installed in the mechanical hand, detecting multipoint within the prescribed work area[2].This will be carried out on the movement of the manipulator trajectory planning. Due to the finished product with high cost, we need to study the kinematics of the robot, and then using the simulation software to simulation.

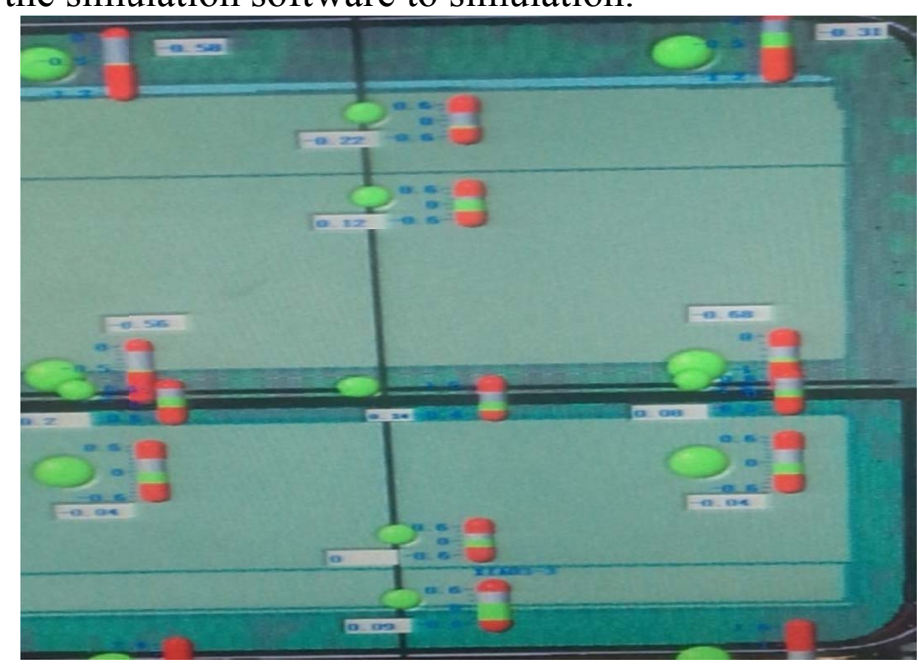

Figure 1: skylight testing point distribution 
Sensor Introduction. As we all know sensors is the important element in automatic detection system, whether sensor performance is good or bad has a direct impact on the performance of the whole test system. The quality of sensor is determined by the performance of the sensor detection system which is an important part of the equipment with high precision and high reliability of sensor detection system engaged in the production and business enterprises[3]. Working process as below

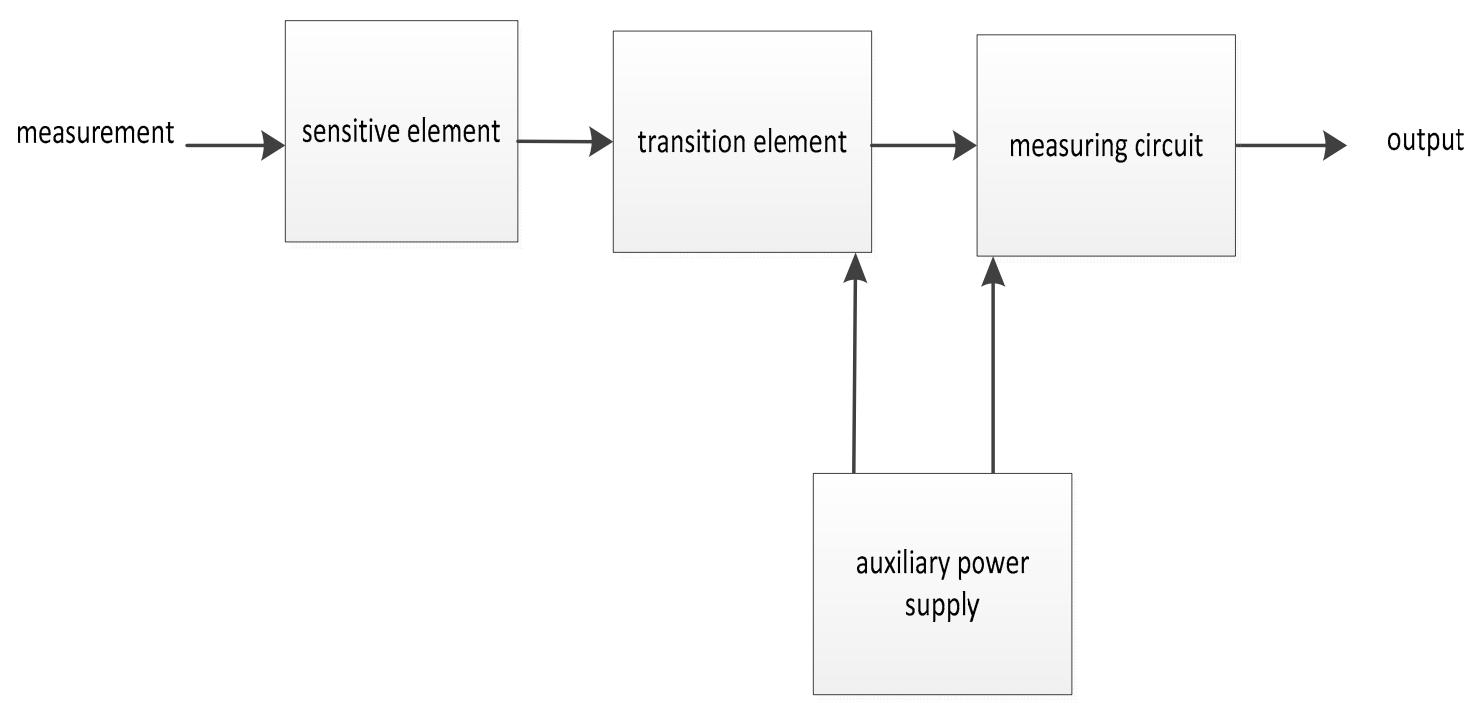

Figure 2 Sensors work flow chart

We use PY-2- C - 010 - type XL0202 distance detection sensor in our study. This kind of sensor parameters as follows: displacement from 10 to $50 \mathrm{~mm}$; Linear accuracy to $\pm 0.1 \%$ of full scale; Reproducibility degree $0.01 \mathrm{~mm}$; Displacement speed can be up to $10 \mathrm{~m} / \mathrm{s}$; Use the trip $10 / 25 / 50$; Displacement force < $4 \mathrm{~N}$; Vibration $5 \sim 2000 \mathrm{HZ}$; The maximum amplitude of $0.75 \mathrm{~mm}(20 \mathrm{~g})$; Vibration $=50 \mathrm{~g}, 11 \mathrm{~ms}$; Impedance allowable error of plus or minus $20 \%$ percentage points; Current cursor $<0.1 \mu \mathrm{A}$; Infinite resolution, without interference from external noise.

\section{Robot Kinematics}

Hand posture and movement study is the focus of robot kinematics. One is positive kinematics problem that is calculated end actuator of the robot posture according to robot each joint angle or displacement; the other is inverse kinematics problem that is calculated each joint Angle or displacement according to the end of the robot pose ${ }^{[4]}$.

Table1 include the specific parameters Simulation data of the robot used in this paper. We calculate the robot inverse kinematics according to these parameters and date here is not specific state the methods to solve the inverse kinematics.

Table 1 robot parameter

\begin{tabular}{|l|l|l|l|l|l|l|l|}
\hline $\mathrm{N}$ & $\theta_{i}$ & $\alpha_{i}$ & $a_{i}$ & $d_{i}$ & $\cos \alpha$ & $\sin \alpha$ & Variable range \\
\hline 1 & $\theta_{1}$ & $-90^{\circ}$ & 0 & 0 & 0 & -1 & $-180 \sim 180$ \\
\hline 2 & $\theta_{2}$ & $0^{\circ}$ & 1150 & 1150 & 1 & 0 & $+76 \sim-60$ \\
\hline 3 & $\theta_{3}$ & $90^{\circ}$ & 0 & 0 & 0 & 1 & $-142.5 \sim+230$ \\
\hline 4 & $\theta_{4}$ & $-90^{\circ}$ & 0 & 1225 & 0 & -1 & $-205 \sim+205$ \\
\hline 5 & $\theta_{5}$ & $90^{\circ}$ & 0 & 0 & 0 & 1 & $-120 \sim+120$ \\
\hline 6 & $\theta_{6}$ & $0^{\circ}$ & 0 & 225 & 1 & 0 & $-200 \sim+200$ \\
\hline
\end{tabular}




\section{ADAMS Main Modules}

Three-Dimension Geometric Model Establishment. Three-dimension geometric modeling usually adopts two methods: one is the use of its own in ADAMS/View module parts library to create a simple parts; The other is in CATIA, Pro/E and UG three-dimensional modeling software to establish the virtual prototype of geometric model into ADAMS/View again [5].

Modeling must follow the principles:

(1)Under the condition of each part has its clear relative movement, we simplify it according to the selected principle of moving pair of reduced, ensure the correctness of the geometric model of each parts movement. In this article, we have six joints, waist joint, shoulder joint, elbow, wrist, hand rotation joint and longitudinal joint. A fixed vice (base with the ground), 5 rotating vice, respectively is the base and the waist joints, waist and big arm joints, arm and forearm joints, the rotation of the forearm and wrist, hand joints and longitudinal joints. Understand the relative motion between all joints, we can make it simplify and then model based on the principle.

(2)When multiple parts relative consolidation can use only one part to replace to reduce the number of motion pair, shorten the kinematic chain length to reduce the calculation error. The robot we used in the mechanical structure is relatively simple, it is needless to expounded detail of each joint internal structure, just need to one which is simplified by each joints to express the parts of a joint movement.

(3)Under the premise try to simplify the robot model, to shorten the modeling time, without affecting the relative motion between the connecting rod. Manipulator each section of the simplified into a rigid body, and express corresponding motion relations, establishing each part of the model and assembly in CATIA into a whole, and prepare for the next step in the ADAMS simulation.

Model Process.(1)We establish its geometric model and assembly in CATIA based on the basic principles of geometric model and automobile skylight each connecting rod structure and basic size detection. Because of our research is robot trajectory of each joint, namely, the change of the angular velocity and angular acceleration of each joint, so guarantee the relative position of the relationship between the correct movement joints in the simplified structure to all parts of each connecting rod and connecting rod consolidation together. Robot object shown in Figure 3, the establishment of the robot motion components shown in Figure 4.

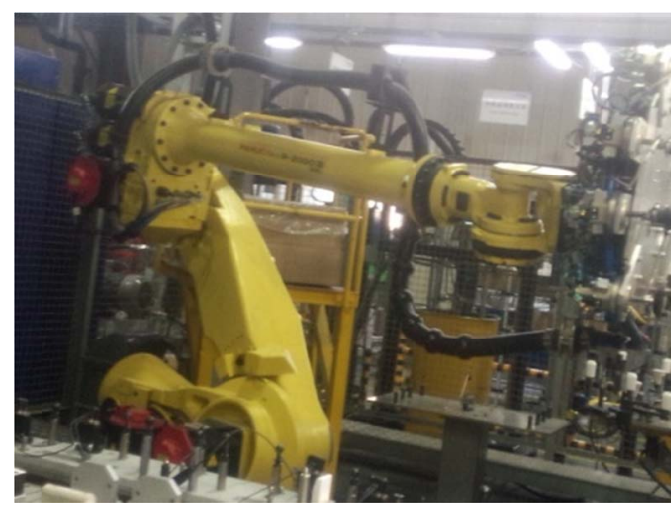

Figure 3 Robot object

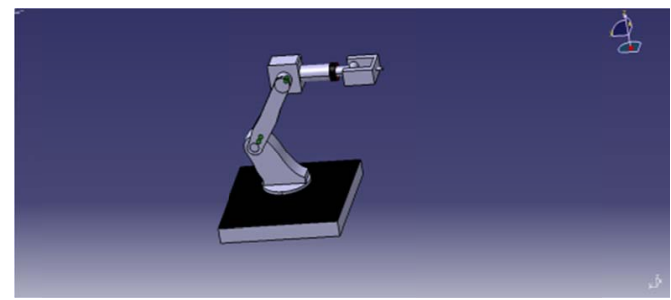

Figure4 robot model

Although there has certain difference between build robots all the moving parts or the overall appearance and the actual car skylight inspection robot, from the perspective of simulation, the difference only influence quality attributes of the robot and the driving force but has no effect on the 
trajectory of each joint, deserve to mention, the simplified model in favor in adding of the constraints, driver and movement parameter measurement.

(2)Enter the View module, set up the working environment, click the File inside the Import button, the imported model needed to Import. Click the OK button, the file can be imported, in this paper, the required import files as shown in Figure 5, imported model as shown in Figure 6.

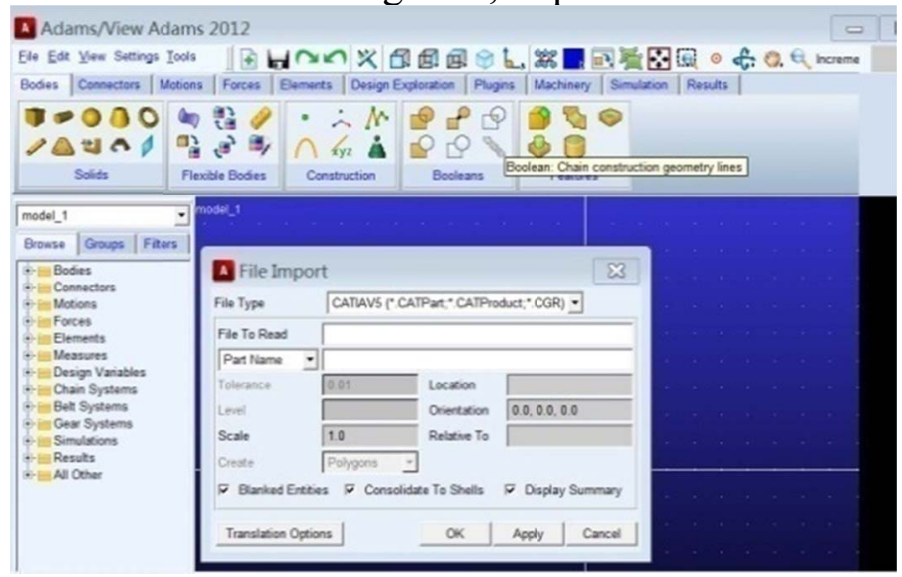

Figure 5 Import file interface diagram

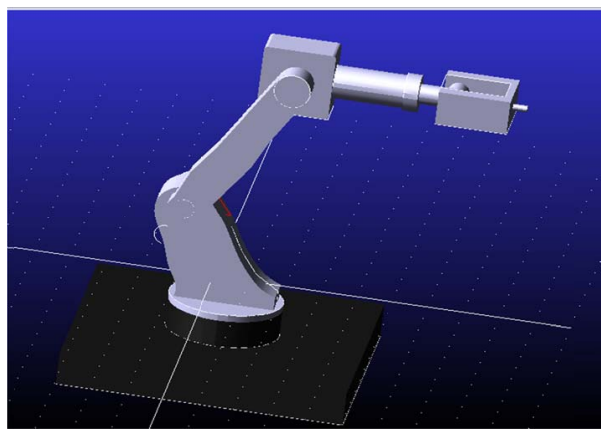

Figure 6 imported model

(3)Add constraints and drive. Including: a fixed (base with the ground); Five revolute(5 joints), the base and the waist joints, waist and big arm joints, arm and forearm joints, the rotation of the forearm and wrist, hand joints and longitudinal joints; Finally impose rotation drive to each motion pair.

(4)Self-checking. Using the Tools menu Model Verify command to check the model before simulation, the information displayed in the "Model verified successfully" means established kinematics model is correct and kinematics simulation analysis can be do. From the information window we can get important information of the model, including the number and types of parts and motion pair, the degrees of freedom of the model are also shown. Figure prototype model test result is shown in Figure 7. 


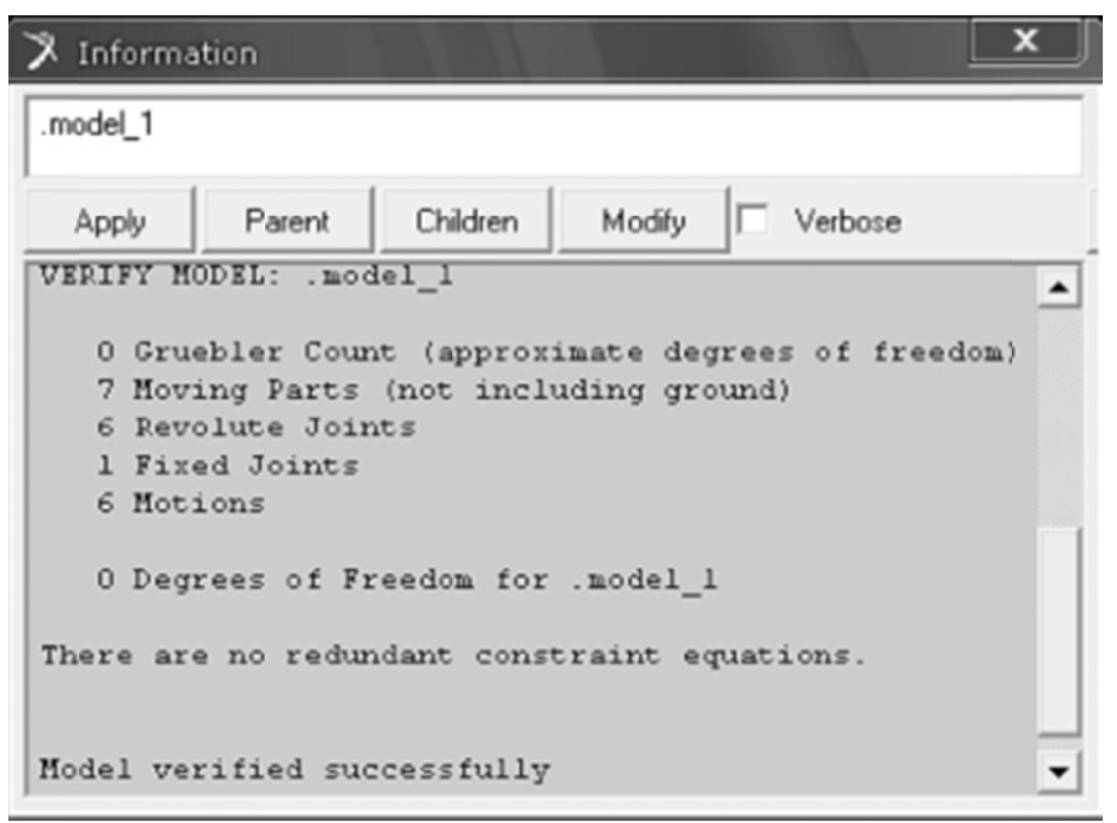

Figure 7 model test result

The six degrees of freedom robot multi-rigid-body model based on ADAMS kinematics analysis. Input function drivers of each joint kinematics positive solutions to ADAMS/View environment simulation: waist joint $30 \mathrm{~d} * \sin (60 \mathrm{~d} *$ time-30d) $+30 \mathrm{~d}$; Shoulder joint $-20 \mathrm{~d} * \sin$ (180d*time-90d)-20d; Elbow joint 20.0d *sin (135d*time-90d) +20d; Wrist back movement-10d*sin (60d* time-30d)-10d; Turn wrist movement 30.0d* $\sin (60 \mathrm{~d} *$ time-30d)+30d; Wrist joints connected sensor $0.1757 t^{5}-2.0683 t^{4}+6.8457 t^{3}-0.0003 t^{2}-0.0002 t+10.0029$. We set up the simulation time $5 \mathrm{~s}$, the simulation step 500.Each joint variable

$\theta_{1} \sim \theta_{6}$ displacement curve as shown in Figure $7 \sim$ Figure11. Hand end point (chuanganqi_cm ) relative to the robot base coordinate system respectively in the $\mathrm{X}, \mathrm{Y}, \mathrm{Z}$ direction of the displacement, velocity and acceleration curve and curve is shown in Figure 12.

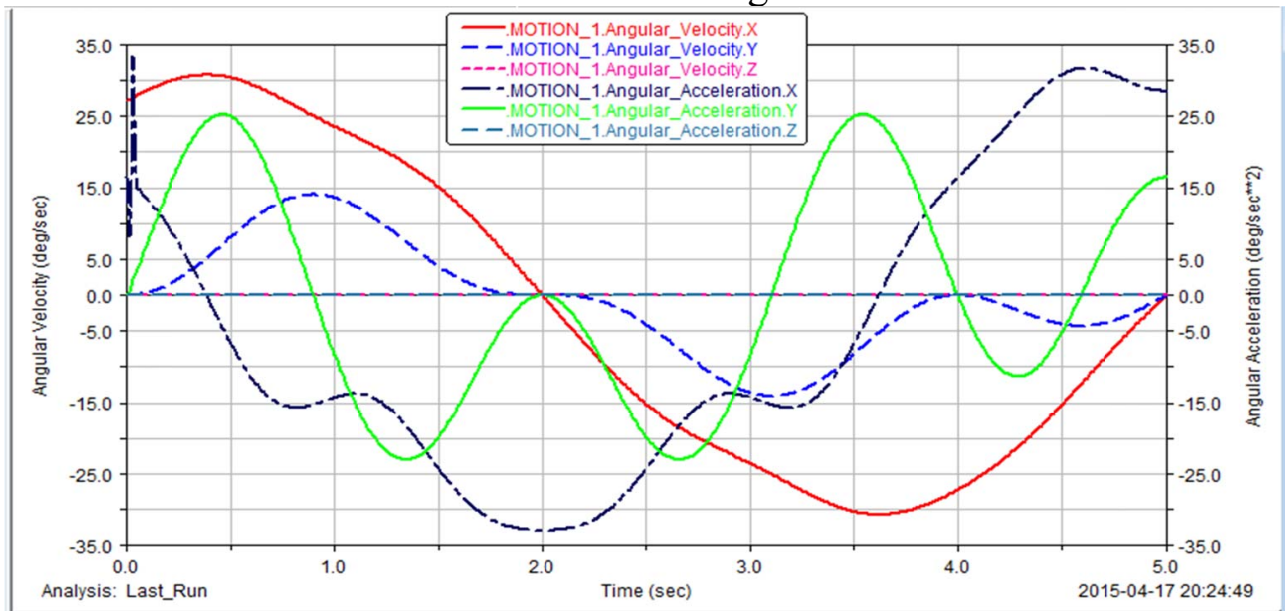

Figure 7 the velocity and acceleration curve of motion 1 


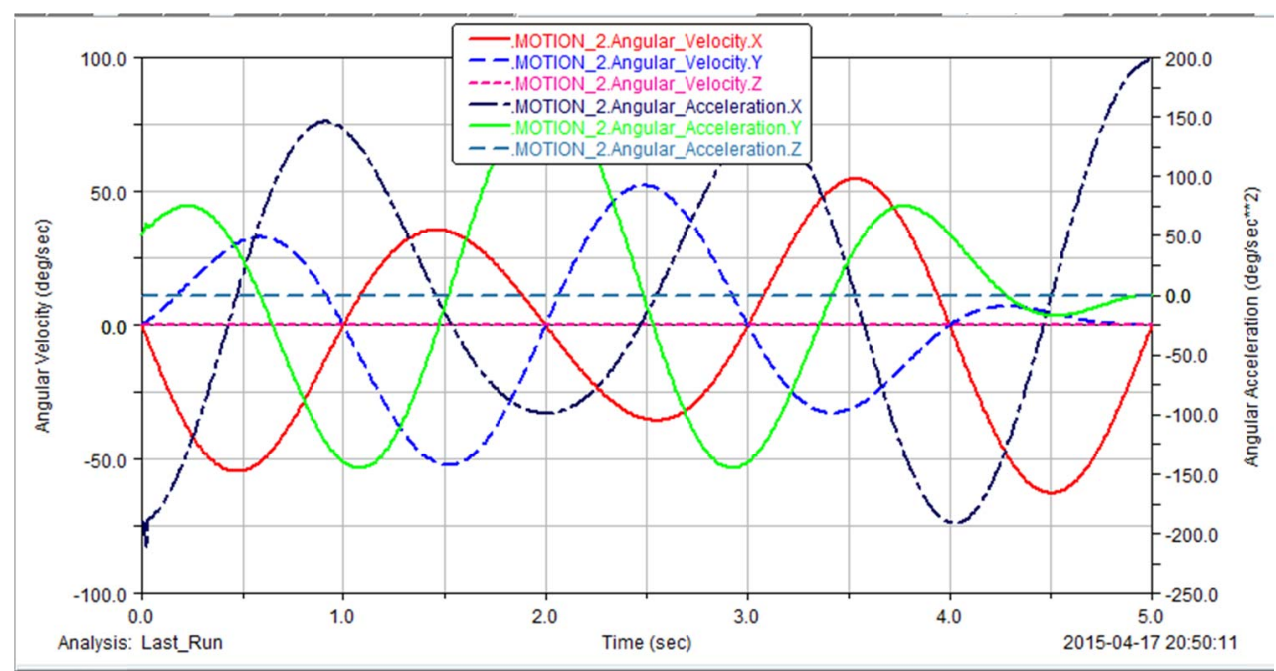

Figure 8 the velocity and acceleration curve of motion 2

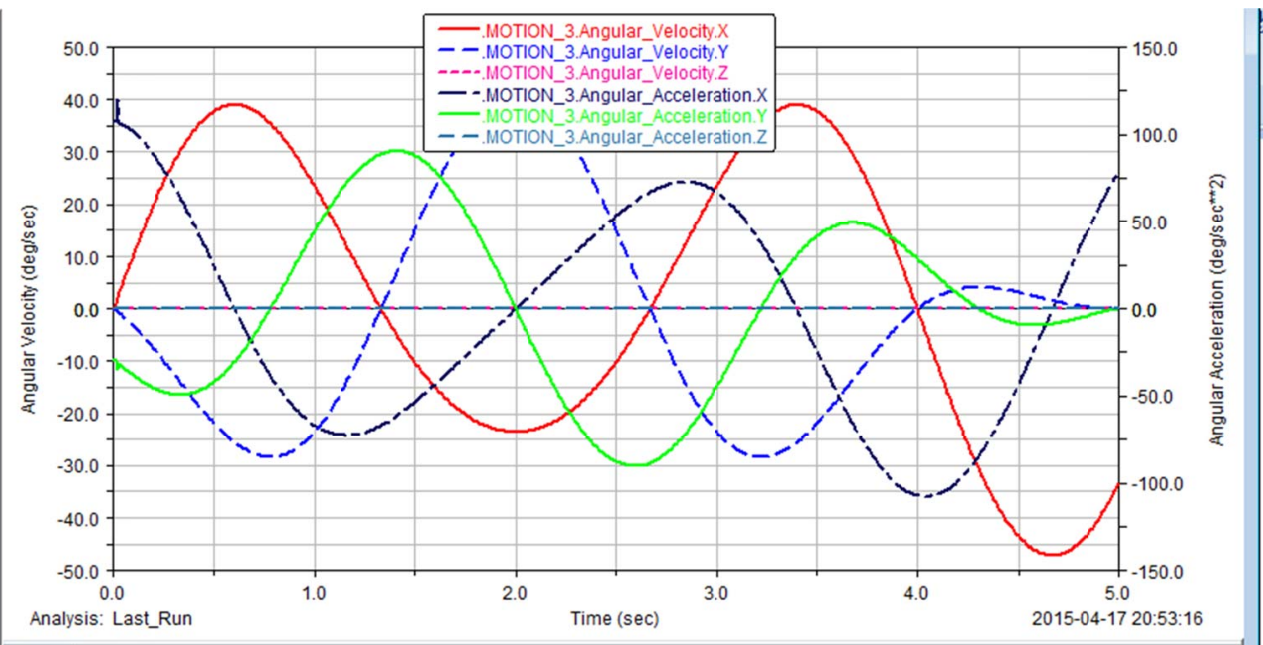

Figure 9 the velocity and acceleration curve of motion 3

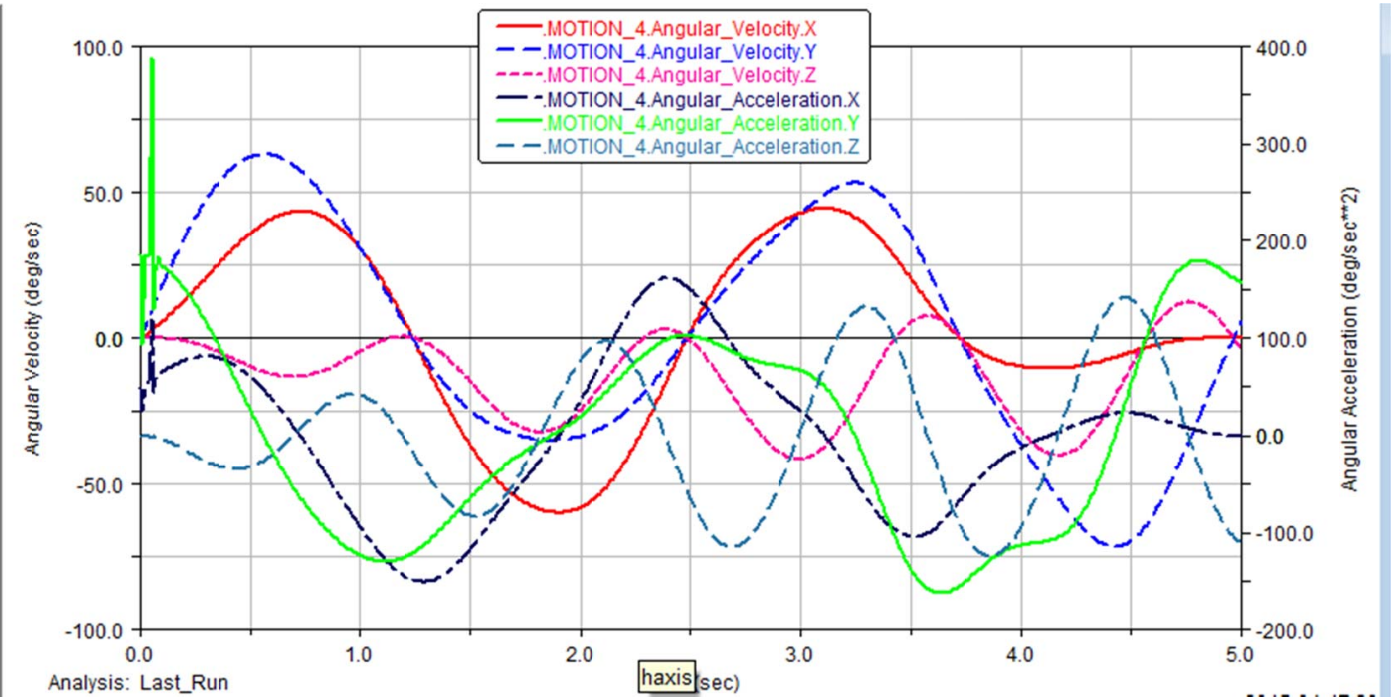

Figure 10 the velocity and acceleration curve of motion 4 


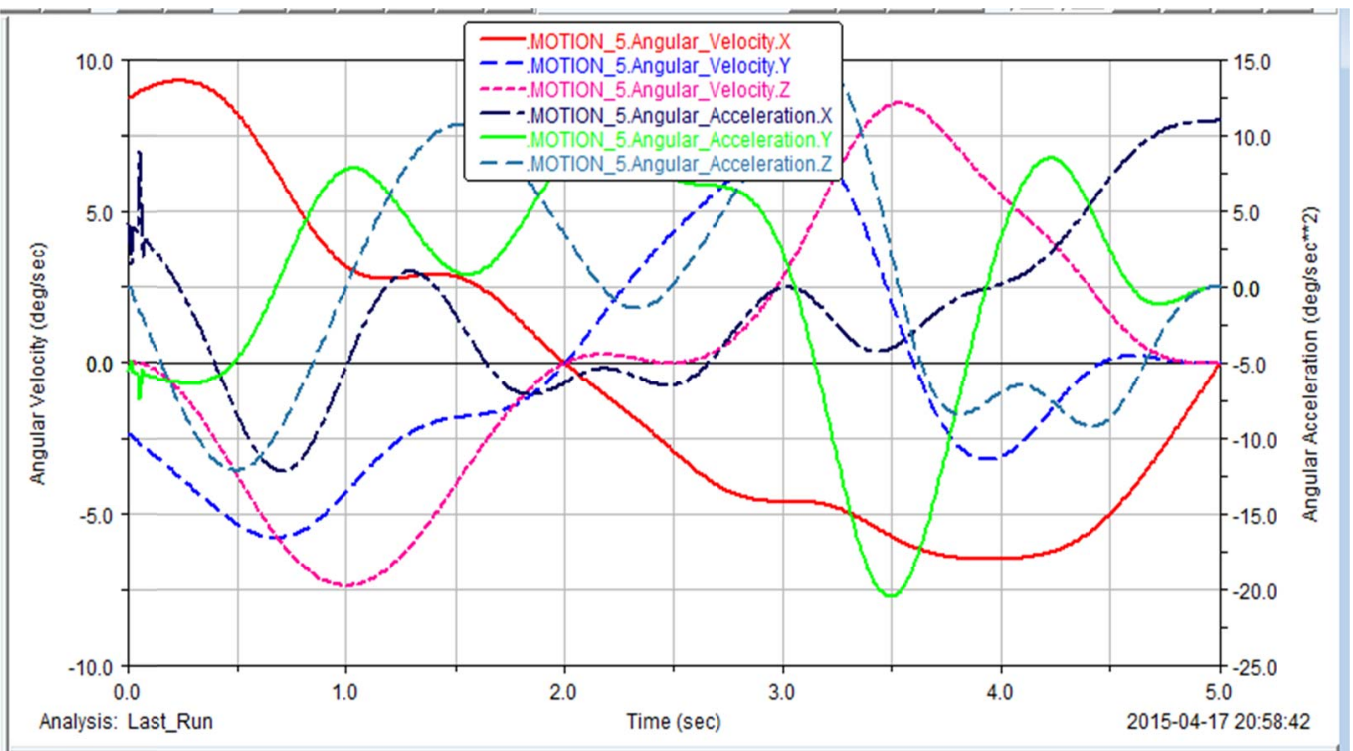

Figure 11 the velocity and acceleration curve of motion5

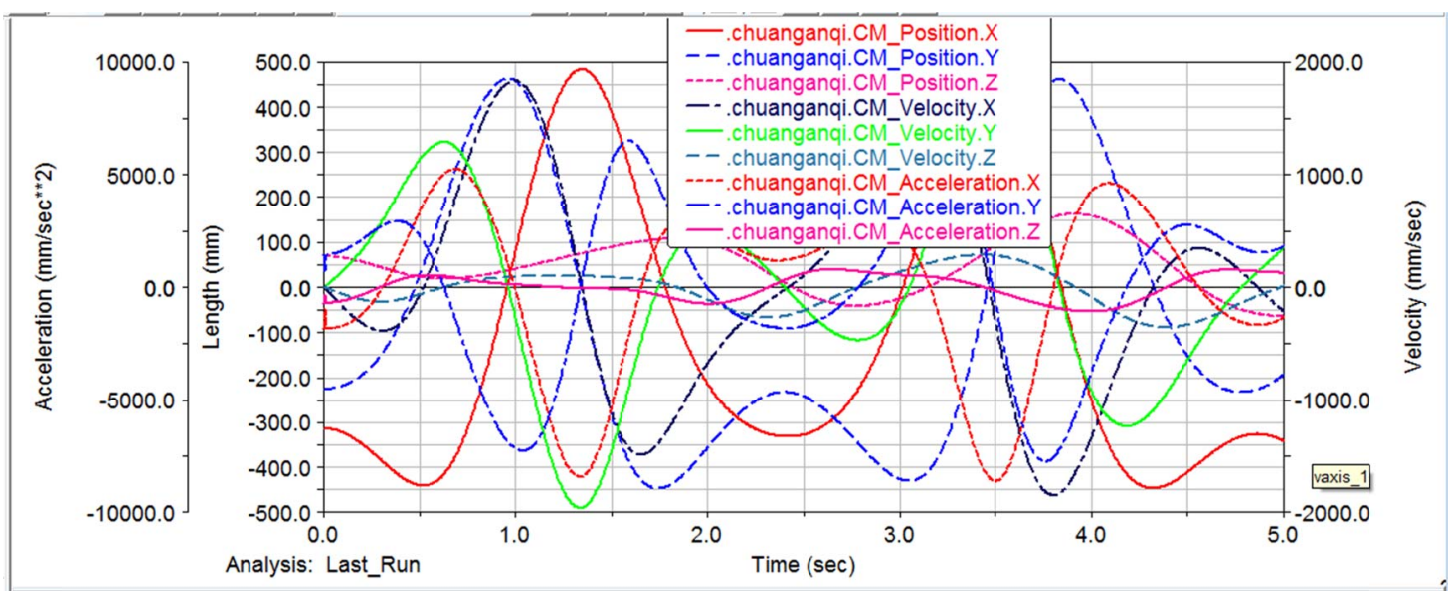

Figure12 sensor center position, velocity, acceleration diagram of Joints 6

Through the Figure $8 \sim 12$ we can see that the first five joint trajectory is smooth, and within the prescribed scope of sports, that movement of the manipulator trajectory is in accordance with our orders and requirements of operation, laid a good groundwork hand sensor below work for us. We watched the Figure 13, through the end point sensor center position, velocity, acceleration curve, we can see the manipulator trajectory continuous and smooth, namely sensor detection process is continuous, and trajectory is smooth. Kinematics simulation Figure shows each joint velocity and acceleration curve in the range of constraints smooth continuous, reached the trajectory planning requirements, the acceleration curve is a smooth transition, effectively solve the problem of robot running stability[6].

\section{Conclusion}

In this article we set up a six degrees of freedom robot joints virtual prototype, and carries on the kinematics simulation analysis research in the basis of the car skylight detection requirements, From the research we can see that ADAMS software itself with powerful measurement and curve analysis ability, so to make the robot kinematics analysis more convenient, quick, and more visual, bring us great convenience. We still need to make further optimization path simulation and improve it. 